\title{
Four quadrant phase mask K-band coronagraphy of NGC 1068 with NAOS-CONICA at VLT
}

\author{
D. Gratadour ${ }^{1,2}$, D. Rouan ${ }^{1}$, A. Boccaletti ${ }^{1}$, P. Riaud ${ }^{1}$, and Y. Clénet ${ }^{3}$ \\ ${ }^{1}$ LESIA, Observatoire de Paris, 5 place Jules Janssen, 92195 Meudon, France \\ e-mail: damien.gratadour@obspm.fr \\ 2 DOTA-ONERA, Avenue de la division Leclerc, 92322 Chatillon, France \\ 3 ESO, Karl-Schwarzschild Strasse 2, 85748 Garching bei München, Germany
}

Received 10 August 2004 / Accepted 1 September 2004

\begin{abstract}
We observed the nucleus of NGC 1068 at $K$ s with NAOS-CONICA on the VLT, using the new four quadrant phase mask coronagraph that we recently developed. As the $K$-band emission is dominated by an intense compact central source, previous $K$-band adaptive optics images were severely affected by the limited exposure time and by scattered light in the immediate vicinity of the source. Thanks to the use of this new type of coronagraph coupled to adaptive optics, the complex dust structure near the central core is becoming observable at a resolution of $0.07^{\prime \prime}$. North of the nucleus, an elongated, bending structure, and a series of four elongated and well aligned knots, superposable on the same pattern previously observed by us at 3.8 and $4.8 \mu \mathrm{m}$ are well identified. This agrees with our first interpretation that, given the direction they align in, these knots may trace shocks induced in the ISM by the passage of the jet, very close to its origin. Moreover, precise relative photometry supports our interpretation of very small dust grains transiently heated by UV photons of the central source. To the South we detect a new group of filamentary structures, distributed in a cone at about $150 \mathrm{pc}$ from the core. They might trace the redshifted southern narrow line region, seen through the dust. Finally, on larger scale (within a radius of three hundred pc) the source has an overall biconical shape whose angle matches well with the bicone observed in the UV-visible.
\end{abstract}

Key words. galaxies: Seyfert - galaxies: individual: NGC 1068 - infrared: galaxies - instrumentation: adaptive optics

\section{Introduction}

NGC 1068, the most studied Seyfert nucleus, is located at an approximate distance of $15 \mathrm{Mpc}\left(1^{\prime \prime} \sim 70 \mathrm{pc}\right)$. Its bright nucleus makes possible Adaptive Optics (hereafter AO) observations in the infrared with very high correction quality. Moreover, this object is considered a classical example supporting the unified model of AGN.

This model (e.g. Krolik 1999) assumes a nuclear source a massive black hole with an accretion disk mainly emitting in the visible, UV and in X-rays - surrounded by a dusty molecular torus which hides the central source from us in type II nuclei. In the case of NGC 1068, observations at high angular resolution (typically $0.1^{\prime \prime}$ ), in all bands from radio to X-ray, show a more complex structure than the simple doughnut picture surrounding the nuclear source. Radio observations reveal a structured jet (Gallimore et al. 1996) and reverberation maser emission probably tracing the inner edges of the obscuring torus (Gallimore et al. 2001), arranged as a rotating disk geometry. A Narrow Line Region (NLR) is seen at UV-visible wavelengths and is structured into high-velocity ionized clouds (Capetti et al. 1995; Cecil et al. 2002). The dusty-molecular environment surrounding this core has also been mapped at different wavelengths. In the IR, elongated dusty structures are detected, N-S $\left(\mathrm{PA} \sim 12^{\circ}\right.$ ), and E-W $\left(\mathrm{PA} \sim 102^{\circ}\right.$ ) (Rouan et al. 1998; Marco \& Alloin 2000) probably tracing the obscuring torus. More recently, AO imaging at a 0.1" resolution (Rouan et al. 2004, hereafter RLG04) revealed the distribution of hot dust in the vicinity of the AGN with a wealth of detail. It put in evidence new structures in the surroundings of the central core, some of them linked to the radio jet, and allowed the cross registration of all maps from radio to UV on a morphological basis, consistently with the absolute registration of Galliano et al. (2003). A recent spectroscopic study with AO (Gratadour et al. 2003) allowed us to put some constraints on the nature of the dust in the putative torus - mainly silicate grains - as well as on its inclination with regard to the line of sight (about $22^{\circ}$ ).

Up to now, reaching a deeper level in brightness was limited by the short exposure time required to avoid saturation. On the other hand, if saturation is deliberately chosen, then the level of scattered light from the central core puts another limit. Coronagraphy is a new way to push these limits further. In this paper, we report on $K$ s-band coronagraphy of the central $12^{\prime \prime}$ region of NGC 1068, and propose some very preliminary interpretations based on simple models. First, we give a short description of the four quadrant phase mask (hereafter FQ-PM) principle and requirements in terms of AO correction as well as of data processing. Then we show the reduced and 
reference subtracted images and give a first interpretation of the new observed structures and photometry of different regions. We give conclusions in the last section.

\section{Observations}

\subsection{The four quadrant phase mask}

The FQ-PM (Rouan et al. 2000) is based on the use of a transmissive phase mask arranged in a four-quadrant pattern: two quadrants on one diagonal providing a $\pi$ phase shift with respect to the two others. The four sub-beams issuing from a bright source located exactly at the center of the mask, assuming an ideal tip-tilt correction, combine in a destructive way, leading to an attenuation factor of up to $12 \mathrm{mag}$ in the lab. A performance evaluation can be found in Riaud et al. (2001, 2003). Since the efficiency of this device in terms of attenuation is related to the quality of the AO correction, and mainly the residual tip-tilt error. Good sky conditions and a high strehl ratio (i.e. more than $40-50$ percent) are mandatory for using such a system on a ground-based telescope.

\subsection{Data acquisition and processing}

Observations have been performed in November 2003 during GTO observations with NAOS-CONICA at the Nasmyth focus of VLT-UT4. The seeing was fairly good $\left(0.7^{\prime \prime}\right)$ and the sky was clear. The AO system was servoed on the central part of NGC 1068, leading to a Strehl ratio at $K$ s ranging from 0.5 to almost 0.7 during the observations. The FQ-PM was first centered on the object and 100 exposures of $1 \mathrm{~s}$ were acquired. Then, after sky acquisition during the same amount of time, a reference star (BD 00413, a G0 star) was centered on the mask, and 50 exposures of $1 \mathrm{~s}$ were recorded. The star has been chosen to reproduce almost the same AO conditions. Strehl ratios obtained with this point source ranged from 0.5 to 0.6 . Images of this reference with about the same AO performance are used to calibrate the effect of imperfect AO loop correction (residual speckles) as well as the residual diffraction pattern of the mask. After sky subtraction, the images are averaged (Fig. 1). The reference was rescaled in intensity and subtracted from the image of NGC 1068 leading to the images of Fig. 2. The noise increase introduced by this subtraction leads us to consider no structures too close to the nucleus, i.e. included within a $0.15^{\prime \prime}(12 \mathrm{pc})$ radius.

\section{Results}

First, on large scales the source has an ovoid shape elongated along PA $\sim 40^{\circ}$, in agreement with previous observations. Note that the diffraction patterns due to the spiders of the telescope, as well as the blind area of the FQ-PM are visible in the image of Fig. 1, and can give the impression of a well defined bicone. Unfortunately, those artifacts are almost aligned with the actual bicone axis, so that the central part of the bicone is not actually observable.

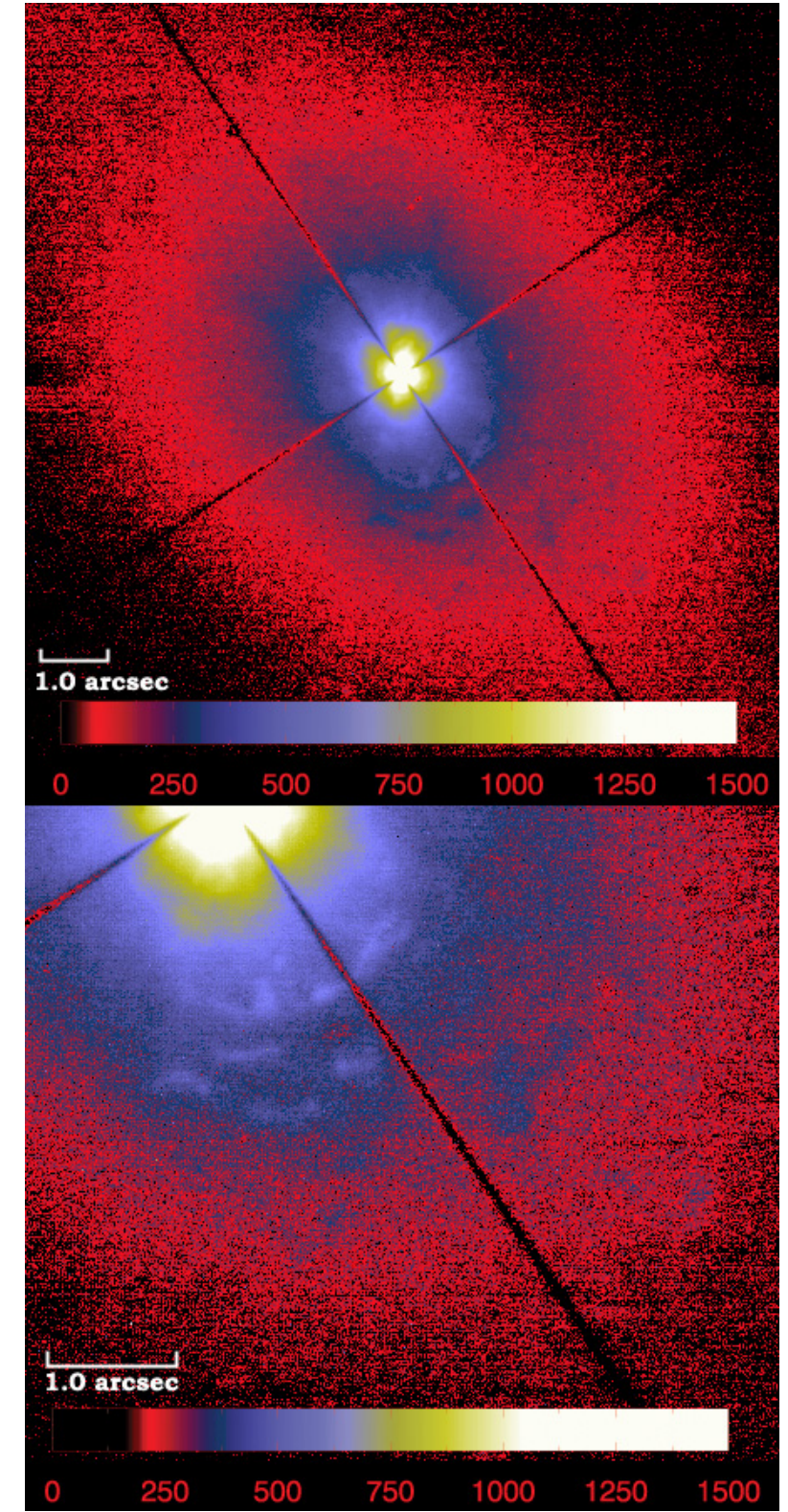

Fig. 1. Coronagraphic image of NGC 1068. A log-scale representation is used for more clarity. North is up and east is left. Top: the whole field: $11.7^{\prime \prime} \times 11.7^{\prime \prime}$, bottom: zoom on filamentary structures south west. The color distribution have been chosen to ensure a good contrast in large scale structures.

\subsection{Northern structures}

North of the nucleus a diffuse emission dominates the images on large scale (Figs. 1 and 2 top, blue and red levels) with a tendency to become more clumpy beyond a radius of a hundred parsecs. Closer to the nucleus, an elongated and bent structure (Fig. 2 bottom, white and yellow levels), along PA $=-10^{\circ}$ is observed, in agreement with the profile of the $K$ s image without mask (RLG04) displayed in Fig. 2 (top). As this structure is clearly affected by the coronagraphic effect, no detailed interpretation of its shape can really be made (see end of Sect. 2), but we suggest that there is an elongated, probably S-shaped structure, crossing the nucleus at PA $=-10^{\circ}$. Directly north 


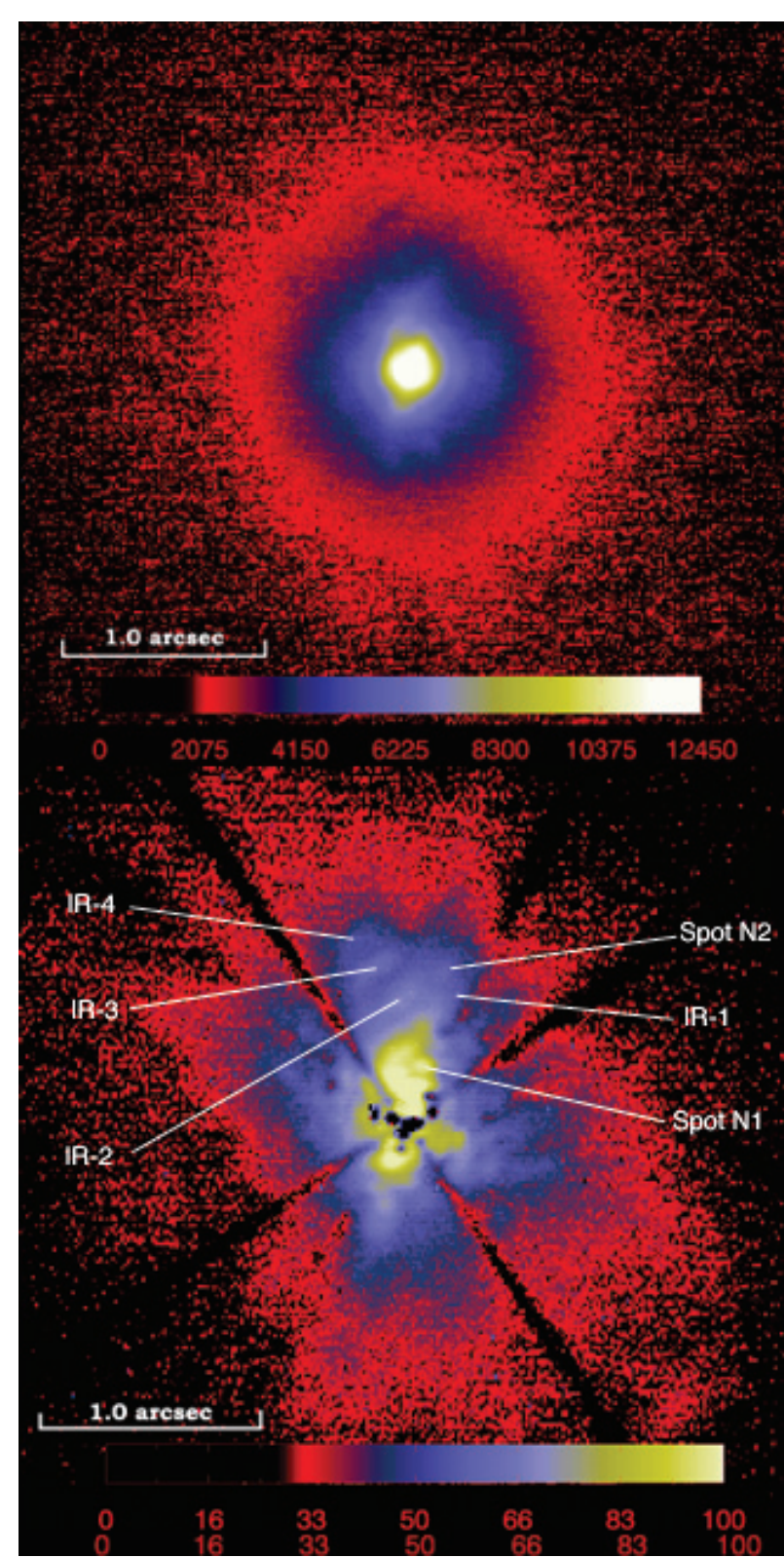

Fig. 2. Top: NGC 1068, Ks band with NACO, without mask. Bottom: reference subtracted coronographic image of NGC 1068 in a log-scale representation. The color distribution has been chosen to ensure a good contrast for structures close to the nucleus.

of this pattern, a new spot is detected which does not correspond to any identified feature. In the following, we will call it spot N1.

Around $15 \mathrm{pc}$ North of the nucleus, an elongated structure with a length of about $20 \mathrm{pc}$ bends clockwise; it looks like a micro-spiral arm, and is identified with the structure depicted in Rouan et al. (1998). Unfortunately, part of this structure is hidden by the blind pattern of the mask. This structure coincides well with cloud C identified first by Evans et al. (1991), if we follow the registration scheme of Galliano et al. (2003) which is consistent with the association of the radio-IR pointlike nucleus with the apex of the optical/UV cone. It is followed by a series of four elongated knots, aligned along PA $=25^{\circ}$, from the North, knots IR-1, IR-2, IR-3 and IR-4) as observed previously in the $L^{\prime}$ and $M$ bands (RLG04). Knot IR-2 coincides with cloud $\mathrm{E}$ of Evans et al. (1991) and knots IR-3 and IR-4 with cloud D. As shown in the top panel of Fig. 2, these structures, especially the four knots, were not clearly detected in previous NAOS-CONICA $K$ s band observations, since the bright central source prevented long exposures. As the radio jet seems to pass through a corridor delineated on one side by these knots, we interpreted them as structures created by hydrodynamic interaction of the jet with the interstellar medium (RLG04). These knots, seen from the $K$ s to the $M$ band, trace hot dust, as revealed by their high color temperature (see Sect. 3.4). Now it is not clear whether they are heated mainly by the central source or by shocks due to the passage of the jet which shapes this elongated structure. Such overdensities bracketing a jet are seen in numerical simulations of jet-cloud interaction in YSOs (de Gouveia Dal Pino 1999). Some authors have shown that such shocks are not very efficient for heating the dust itself (Bock et al. 2000), but Kraemer \& Crenshaw (2000) have shown that in the Narrow Line Region (NLR), photoionization by the central source has to be combined with another ionizing mechanism such as UV or X-ray emission generated by shocks. This is required up to about one hundred pc from the nucleus. We will however consider in the following, as a first order estimate, that those regions, even if shaped by the passage of the jet, are mainly heated by the central engine radiation. We also do not consider the effect of screening material between the central source and these knots. Note the presence of a new spot, between the first and the second knot. This spot seems unresolved, and no elongation is observed. We will call it spot $\mathrm{N} 2$ in the following.

To compare these data to the distribution of gas in the vicinity of the central core, we superimposed our coronagraphic image on narrow band (NB) images of Thompson et al. (2001), according to the registration scheme previously cited. Most of the structures have a counterpart in the HST image, except knot IR-1, and spots N1 and N2. It appears that in most of these knots the dust distribution is coincides with gas patches seen in the HST NB images. However, with our coronagraphic image the resolution is pushed further, clearly showing substructures, as for instance in cloud D.

\subsection{Southern structures}

First, close to the nucleus and to the South of it, a peculiar structure, identified in the $L^{\prime}$ and $M$ bands, called south tail in RLG04 and associated to the southern structure of Bock et al. (2000), is clearly detected in the reference-subtracted image and can be very well superimposed in the pattern detected in thermal IR.

At a larger distance from the nucleus and to the Southwest of it, totally new features, i.e. not seen in the $K$ s image without mask, appear in Fig. 1, south-West of it: a shell-like distribution of clumpy emitting regions around the well-known ionization cone axis. They are comparable in shape and extension to the N-E knots but they are sharper and more distant from the nucleus, so that a similar interpretation cannot be given. They may be the Southern counterpart in the ionization bicone of 
the filamentary clouds observed north of the nucleus in HST UV-visible images (Capetti et al. 1995). Part of this structured group of clouds, appearing as a set of four arclets, about $160 \mathrm{pc}$ south of the central central core (Fig. 1 bottom) is unfortunately hidden by the blind pattern of the mask. At almost the same distance, but Southwest, we observe a more patchy emission, which could belong to the same set of structures. Thompson et al. (2001) argued that the Southern ionization cone should be more conspicuous in IR due to extinction by the galactic plane of NGC 1068. Moreover, south of the nucleus, structured features appear in $\mathrm{H} \alpha$ and Paschen $\alpha$ images beyond $150 \mathrm{pc}$ that have not been interpreted yet. These features have a more irregular morphology than the arclets detected in our coronagraphic image and extend much closer to the nucleus. A patchy but not very structured emission also appears also on their [Si VI] image. As discussed by the authors, this coincidence between $\mathrm{H} \alpha$ and dust emission is comparable to what happens in galactic HII regions, and could be due to dust entrained into HII regions. Nevertheless, these features are clearly not detected in Mid-IR either by Bock et al. (2000) or by Tomono et al. (2001). An explanation could be the lower sensitivity of the $10 \mu \mathrm{m}$ observations. These structures are showing up in $\mathrm{H} \alpha$ where the lack of a powerful central source allows a better sensitivity to surrounding structures. In the $K$ s band, without mask, these structures were hidden by the scattered light from the central source; the same kind of effect can corrupt the mid-IR data.

Such a structured morphology suggests a very peculiar mechanism in this NLR. One interpretation could be that is the effect of the AGN pulsating wind (Elvis 2000) that could produce those regular arclets. Another explanation could be hot gas from supernovae, flowing out in parsec-scale bubbles from a central starburst region surrounding the central black hole. Spectroscopy of these arclets could give more clues as to their real nature. Note that these arclets are distributed on the outer edge (around $160 \mathrm{pc}$ ) of the structured feature observed in $\mathrm{H} \alpha$. Moreover, in the corresponding Northern part of the ionization cone, i.e. 150 pc Northwest, Kraemer \& Crenshaw (2000) found decelerating clouds and evidence for shock-generated UV-X-ray emission. So the arclets could trace the interface between the outflowing gas and the interstellar medium, where the strength of emission lines increases, even if comparison to photoionization models has shown (Kraemer \& Crenshaw 2000) that photoionization could be enough to explain the observed emission.

\subsection{East-West complex}

In the reference subtracted image of Fig. 2, a complex east-west structure appears with a prominent western part. Close to the core the structure, identified by Rouan et al. (1998) as a probable trace of the molecular torus, is not clearly detected. The pattern we detect (Fig. 2 bottom), crossing the nucleus, seems partly corrupted by reference subtraction, and any interpretation is difficult because of its complex shape. The $K$ s image without mask revealed only an elongated structure at a very low level along PA $=102^{\circ}$ as shown in Fig. 2 (top), which is clearly not comparable to coronagraphic images. No relative
Table 1. Photometry and $K s-L^{\prime}$ color ratios of several regions around the central source. The error in estimated magnitude is $\pm 0.3 \mathrm{mag}$, and the aperture radius is given in ".

\begin{tabular}{cccc}
\hline \hline Pos & Ap Rad & Mag & $K s-L^{\prime}$ \\
\hline knot IR-1 & 0.05 & 14.0 & 4.0 \\
knot IR-2 & 0.05 & 14.2 & 4.2 \\
knot IR-3 & 0.05 & 14.2 & 4.6 \\
knot IR-4 & 0.05 & 15.0 & 4.5 \\
\hline Tail & 0.05 & 13.9 & 4.3 \\
Spot N2 & 0.04 & 16.0 & - \\
\hline
\end{tabular}

photometry can be performed since we found no clear corresponding structures in the $L^{\prime}$ and $M$ bands.

\subsection{Photometry}

We performed absolute photometry on the sky-subtracted image (Fig. 1), using the NAOS-CONICA zero point magnitude estimated during our observing night, scaled with the mean airmass during the observations. We used $L^{\prime}$ measurements from data presented in RLG04. The extinction of the coronagraph in the center prevents photometric measurements too close to the nucleus. Therefore photometry of Spot N1 and elongated structures close to or crossing the central source are not presented in Table 1. The color found for knots IR-1, IR-2, IR-3 corresponds to dust at around $500 \mathrm{~K}$ and for knot IR-4 and the southern tail, at $520 \mathrm{~K}$, i.e. a temperature fully consistent with those derived from the $L^{\prime}-M$ color index (RLG04). In a simple radiative transfer model, we can assume that the emission from the central engine is the main source of excitation for these knots, very close (less than $80 \mathrm{pc}$ from the nucleus, i.e. within $1.0^{\prime \prime}$ ) to it. The color indices given by this simple model imply temperatures for classical grains below $400 \mathrm{~K}$. Any dust in the line of sight between the knots and the central source would even reduce this temperature. This scheme thus favores the interpretation that we proposed in RLG04, where very small dust grains (VSG) would be responsible for most of the emission in the knots. Transient heating of VSG (as depicted in Desert et al. 1990 ) is able to explain a high color temperature. The nature of those VSG is still a matter of debate, but the strong UV field probably coupled with the presence of shock fronts due to the interaction of the jet with the ISM would likely destroy silicate VSG. On the other hand, nanodiamonds are known to form in such conditions and are able to survive in such an X-ray/UV radiation field. This is why we proposed them as the best candidate to explain these high color ratio. In a recent paper Mason et al. (2004) found no evidence for anomalous grain distribution in all radial directions, but they used lower spatial resolution, with seeing-limited observations on a $3.8 \mathrm{~m}$ telescope through a $0.45^{\prime \prime}$ slit. Their observations are so corrupted, and probably dominated, by regions beyond our location of interest, closer to the nucleus (for instance the structure coincident with cloud C). In this study we reached almost $0.05^{\prime \prime}$ in spatial resolution and the region that we believe to be dominated by VSG emission is very restricted (up to $15 \mathrm{pc}$, i.e. $0.2^{\prime \prime}$ ). 
Finally, Kraemer \& Crenshaw (2000) showed that shockgenerated UV-X-ray emission cannot be neglected, but this occurs at a larger distance from the nucleus (more than $100 \mathrm{pc}$ ), where the density of the interstellar medium seems to increase as the photoionizing continuum decreases. Shock-generated UV-X-ray emission could thus be a significant source of excitation for these knots, especially close to cloud $\mathrm{C}$, known to be a shock interface, where the bending of the radio jet is commonly interpreted as interaction of the jet with a giant molecular cloud. Nevertheless there is no definite evidence in favor of this interpretation, and our simple model, with transiently heated VSG is able by itself to reproduce these high color temperatures. A more comprehensive study will be done using deconvolved data from RLG04 and overall superposition with both coronagraphic and multi-wavelength data in a future paper. Moreover, precise thermal IR spectroscopy of this faint region compared to the surrounding structures, with this resolution, is essential to confirm the VSG hypothesis.

\section{Conclusion}

Coronagraphy coupled to AO is a totally new approach to studying the close environment of the core of nearby AGN, and to look for new structures that would otherwise be hidden by the PSF of the core. We used a new type of coronagraph with NAOS-CONICA at $K$ s to image the central part the archetypal Seyfert II nucleus NGC 1068. These observations show a complex environment more structured closer to the nucleus than previously imaged in this band. The identified structures are similar to what was observed previously at longer wavelengths (3.8 and $4.8 \mu \mathrm{m}$ ), at the same resolution without mask; and up to now they were totally hidden by the dominating emission of the nucleus at $K \mathrm{~s}$. Shape and photometry are in very good agreement with the previous interpretation of elongated knots, shaped by the passage of a jet, and composed of very small dust grains, transiently heated by the central engine. Moreover, some new structures are detected very close to the nucleus (a few tenth of pc) at very low levels. New filamentary structures in a region South of the nucleus, that could be the counterpart of the northern ionizing cone, have been detected. These coronagraphic images have been taken as a feasibility test and we spent only a hundred of seconds of integration on the source. However the results obtained agree entirely with our first observations in the Near and thermal IR and so confirm the benefit from the use of a FQ-PM with AO to study the surrounding structures of the cores of AGNs.

\section{References}

Bock, J. J., Neugebauer, G., Matthews, K., et al. 2000, AJ, 120, 2904 Capetti, A., Macchetto, F., Axon, D. J., Sparks, W. B., \& Boksenberg, A. 1995, ApJ, 452, L87

Cecil, G., Dopita, M. A., Groves, B., et al. 2002, ApJ, 568, 627

de Gouveia Dal Pino, E. M. 1999, ApJ, 526, 862

Desert, F.-X., Boulanger, F., \& Puget, J. L. 1990, A\&A, 237, 215

Elvis, M. 2000, ApJ, 545, 63

Evans, I. N., Ford, H. C., Kinney, A. L., et al. 1991, ApJ, 369, L27

Galliano, E., Alloin, D., Granato, G. L., \& Villar-Martín, M. 2003, A\&A, 412, 615

Gallimore, J. F., Baum, S. A., \& O’Dea, C. P. 1996, ApJ, 464, 198

Gallimore, J. F., Henkel, C., Baum, S. A., et al. 2001, ApJ, 556, 694

Gratadour, D., Clénet, Y., Rouan, D., Lai, O., \& Forveille, T. 2003, A\&A, 411, 335

Kraemer, S. B., \& Crenshaw, D. M. 2000, ApJ, 544, 763

Krolik, J. H. 1999, Active galactic nuclei (Princeton Univ Press)

Marco, O., \& Alloin, D. 2000, A\&A, 353, 465

Mason, R. E., Wright, G., Pendleton, Y., \& Adamson, A. 2004, ArXiv Astrophysics e-prints

Riaud, P., Boccaletti, A., Baudrand, J., \& Rouan, D. 2003, PASP, 115, 712

Riaud, P., Boccaletti, A., Rouan, D., Lemarquis, F., \& Labeyrie, A. 2001, PASP, 113, 1145

Rouan, D., Lacombe, F., Gendron, E., et al. 2004, A\&A, 417, L1

Rouan, D., Riaud, P., Boccaletti, A., Clénet, Y., \& Labeyrie, A. 2000, PASP, 112, 1479

Rouan, D., Rigaut, F., Alloin, D., et al. 1998, A\&A, 339, 687

Thompson, R. I., Chary, R., Corbin, M. R., \& Epps, H. 2001, ApJ, 558, L97

Tomono, D., Doi, Y., Usuda, T., \& Nishimura, T. 2001, ApJ, 557, 637 